\title{
The p53 codon 72 polymorphism is associated with risk and early onset of breast cancer among Saudi women
}

\author{
ABEER AL-QASEM ${ }^{1}$, MOHAMED TOULIMAT $^{2}$, ASMA TULBAH $^{2}$, NASER ELKUM $^{3,5}$, \\ TAHER AL-TWEIGERI ${ }^{4}$ and ABDELILAH ABOUSSEKHRA ${ }^{1}$
}

\begin{abstract}
Departments of ${ }^{1}$ Biological and Medical Research, ${ }^{2}$ Pathology, and ${ }^{3}$ Biostatistics, Epidemiology and Scientific Computing;
${ }^{4}$ Oncology Center, King Faisal Specialist Hospital and Research Center, Riyadh 11211, KSA
\end{abstract}

Received September 22, 2011; Accepted December 15, 2011

DOI: $10.3892 / \mathrm{ol} .2012 .581$

\begin{abstract}
Breast cancer has a major impact on the health of women worldwide. In the Kingdom of Saudi Arabia (KSA), breast cancer incidence is on the increase and is characterized by early onset and aggressiveness. Owing to the importance of the TP53 gene in breast carcinogenesis, we analyzed the possible link between TP53 single nucleotide polymorphisms (SNPs) and the risk of breast cancer in Saudi women by direct sequencing of the TP53 gene exon 4 from 100 breast cancer tissues. The proportion of the polymorphic forms of SNP72 in the Saudi breast cancer patients were: Arg/Arg (RR), 39\%; Pro/Pro (PP), 36\%; and Arg/Pro (RP), 25\%. The frequencies of these forms in disease-free Saudi women were 7.59, 22.22 and $60.19 \%$, respectively. This indicates that the RR form of the codon 72 polymorphism is a potential risk factor, whereas the RP form is a protection factor against breast cancer among Saudi women $(\mathrm{p}=0.0001)$. Moreover, the results have shown that the p53 R72P SNP is significantly associated with the early onset of breast cancer in the Saudi population $(p=0.0138)$. However, the codon 47 polymorphism appears to have no role in this disease among Saudi women. These results indicate that the TP53 gene could play a major role in breast carcinogenesis and the early onset of the disease among Saudi women.
\end{abstract}

\section{Introduction}

Breast cancer is the most common malignancy and the leading cause of cancer mortality among women, accounting for $23 \%$ of the total cancer cases and $14 \%$ of the cancer mortalities

Correspondence to: Dr Abdelilah Aboussekhra, King Faisal Specialist Hospital and Research Center, BMR, MBC \#03-66, P.O. Box 3354, Riyadh 11211, KSA

E-mail: aboussekhra@kfshrc.edu.sa

Present address: ${ }^{5}$ Dasman Centre for Research and Treatment of Diabetes, P.O. Box 1180, Dasman 15462, Kuwait

Key words: p53, SNP72, breast cancer, early onset worldwide in 2008 (1). In the Saudi population, which is young with more than $50 \%$ aged less than 20 years, most of the breast cancer cases are diagnosed at an early age (2). According to the 2002 annual report of the Saudi National Cancer Registry, breast cancers that developed prior to the age of 40 comprise $26.4 \%$ of all female breast cancers, compared with $6.5 \%$ in the USA. It has been revealed that being aged 40 years or younger is an independent risk factor for relapse in operable Saudi breast cancer patients, indicating that early onset is a serious clinical problem in this population (3). Different types of genetic and epigenetic alterations as well as polymorphisms are significant in the stepwise development of breast neoplasms. The TP53 gene is the most frequently altered gene in human cancer, including breast carcinoma (4). This tumor suppressor gene is a multifunctional tetrameric transcription factor involved in the control of cell cycle progression, DNA repair, apoptosis and senescence (5).

In addition to genetic mutations, breast cancer prognosis may also be affected by TP53 single nucleotide polymorphisms (SNPs) that are involved in DNA damage control and repair. Among the polymorphisms reported in the TP53 coding region, only two alter the amino acid sequence; SNPs 72 and 47 (6-7). The codon 72 polymorphism, the most common SNP in the TP53 gene, occurs in the non-conserved proline-rich region of exon 4 (8-9). This polymorphism encodes either arginine (CGC) or proline (CCC) leading to three variant forms: Arg/Arg, Arg/Pro and Pro/Pro (RR, RP and PP, respectively) (9-10). A meta-analysis has revealed statistically significant differences in the frequencies of the two alleles among healthy individuals (the frequency of the Pro allele is $17 \%$ in Sweden and $63 \%$ in Nigeria) (8) and among ethnic groups (the Pro allele frequency is approximately $60 \%$ in African Americans, but $30-35 \%$ in Caucasian Americans) (11). Previous studies have shown that this common polymorphism is involved in modulating p53 apoptotic function, and as such it may affect the response to chemotherapy (9).

The codon 47 polymorphism, a rare TP53 SNP also located in exon 4, substitutes the wild-type proline (CCG) with serine (TCG) in a small subset of individuals $(8,11)$. The frequency of this polymorphism also varies among ethnic groups (the allele frequency is approximately $5 \%$ in African Americans, while it is not found in Caucasian Americans) (12). Findings of previous studies have demonstrated that, in addition to the 
Table I. Frequencies of the TP53 codon 72 polymorphic forms among Saudi breast cancer patients and a control group.

\begin{tabular}{lccccr}
\hline & & \multicolumn{3}{c}{ SNP72 polymorphic forms } & \\
\cline { 3 - 5 } Sample type & Total cases & RP $(\%)$ & PP $(\%)$ & RR $(\%)$ & p-value \\
\hline Control & 108 & $65(60.19)$ & $24(22.22)$ & $19(17.59)$ & $<0.0001$ \\
Patient & 100 & $25(25)$ & $36(36)$ & $39(39)$ & \\
\hline
\end{tabular}

SNP, single nucleotide polymorphism; RP, Arg/Pro; PP, Pro/Pro; RR, Arg/Arg.

R72P SNP, the codon 47 polymorphism is also functionally significant due to its importance in the transactivation of the pro-apoptotic target genes of the p53 protein (13). Therefore, the p53 P47S SNP may influence the risk and progression of cancer and the efficiency of therapy $(6,13)$.

In the present study, we investigated the possible impact of the codon 72 and 47 polymorphisms on breast cancer risk and the early onset of this disease among Saudi women.

p53 is a tumor suppressor gene that plays a significant roles in the etiology of breast cancer. In addition to mutations, several SNPs have also been revealed to modulate p53 function and are therefore thought to be risk factors for numerous types of cancer, including mammary carcinomas.

\section{Materials and methods}

Samples. A total of 100 invasive ductal carcinoma tumor tissues were collected from Saudi women. The experimental protocol was approved by the Institutional Basic Research and Ethics Protocol Committees (RAC proposal no. 2040037), and signed informed consent was obtained from all the enrolled patients. The age of these patients at the time of diagnosis ranged from 22 to 80 years (median, 51 years). As controls, 108 fresh blood samples $(5 \mathrm{ml})$ were collected from healthy Saudi women. The age of the controls ranged from 17 to 76 years (median, 46.5 years).

Ten tissue sections of $30 \mu \mathrm{m}$ were collected from each breast cancer tissue sample using Microm HM-550 and transferred to a $1.5 \mathrm{ml}$ microtube. The collected breast tumor samples were then stored at $-80^{\circ} \mathrm{C}$ until required for analysis The blood samples were analyzed immediately following collection.

DNA amplification and sequencing. Genomic DNA was purified using the Gentra Puregen kit (Gentra Puregen for blood and tissue kit, cat. no D-50K1-4) according to the manufacturer's instructions. Standard PCR was performed to amplify exon 4 of the TP53 gene, using the HotStar Taq polymerase kit (Qiagen, Chatsworth, CA, USA). The primer set used for this amplification was: forward, 5'-TGA GGA CCT GGT CCT CTG AC-3'; and reverse, 5'-CGG CCA GGC ATT GAA GTC TC-3'. Each PCR reaction was preformed in a total volume of $25 \mu \mathrm{l}$ containing 4 ng genomic DNA, 0.04 units Taq DNA polymerase, $0.5 \mathrm{mM}$ dNTPs, $1 \mathrm{mM}$ primers and $1.5 \mathrm{mM} \mathrm{MgCl}_{2}$. A 370-bp fragment was amplified using a PCR program starting with a denaturation step of $10 \mathrm{~min}$ at $94^{\circ} \mathrm{C}$, followed by 35 cycles of $45 \mathrm{sec}$ at $94^{\circ} \mathrm{C}, 45 \mathrm{sec}$ at $62^{\circ} \mathrm{C}, 45 \mathrm{sec}$ at $72^{\circ} \mathrm{C}$ and followed by a final extension step of $10 \mathrm{~min}$ at $72^{\circ} \mathrm{C}$. The PCR products were directly sequenced using ABI Prism BigDye Terminator v3.1 cycle sequencing kit. Asymmetric PCR was performed as described for the standard PCR, with either the forward or reverse primer. The unincorporated dye-labeled terminators were removed using the DyeEx 96 kit. Finally, the reaction product was resuspended in a formamide loading buffer and then separated and detected in the ABI $3730 \times 1$ DNA analyzer (Applied Biosystems, Foster City, CA, USA). The analysis of the obtained sequence was performed using the GeneBank database, NT_010718.

Statistical analysis. Statistical analysis was carried out using the SPSS program version 17 and the Chi-square test. $\mathrm{P} \leq 0.05$ was considered to indicate a statistically significant result.

\section{Results}

p53 codon 72 genotype is associated with a risk of breast cancer. The p53 codon 72 genotype was determined by sequencing exon 4 from 100 breast cancer tissues and 108 blood samples from healthy women. Table I shows that the proportion of the polymorphic forms of SNP72 in the Saudi breast cancer patients were: RR, 39\%; PP, 36\%; and RP, $25 \%$. The frequencies of these forms in disease-free Saudi women were $17.59,22.22$ and $60.19 \%$, respectively. This result shows that the RR genotype is more prevalent in breast cancer patients, while the RP genotype is more abundant among normal controls. However, allele frequencies did not differ significantly between the patients and the controls. The Arg allele frequency was 47.7 and $51.1 \%$ in the controls and the patients, respectively, whereas the frequencies of the corresponding Pro allele were 52 and $48.5 \%$, respectively.

A statistically significant association was found between SNP72 and risk of breast cancer $(\mathrm{p}=0.0001)$. The RP genotype had an association with protection against breast cancer, whereas the RR genotype was associated with breast cancer in the Saudi population (Table I).

Association between p53 codon 72 polymorphism and the age of Saudi breast cancer patients. To investigate whether the TP53 codon 72 polymorphism is associated with the early onset of breast cancer in Saudi women, the age of the patients was compared with the polymorphic forms of SNP72 (Table II). The RR genotype was more prevalent in older breast cancer patients ( $\leq 50$ years) as compared with young patients (>50 years), with 53.1 and $33.3 \%$, respectively. The RP 
Table II. Association between the TP53 codon 72 polymorphic forms and the age of Saudi breast cancer patients.

\begin{tabular}{lccccc}
\hline \multirow{2}{*}{$\begin{array}{l}\text { Age group } \\
\text { (years) }\end{array}$} & $\begin{array}{c}\text { Number of } \\
\text { patients }(\%)\end{array}$ & RR $(\%)$ & RP $(\%)$ & PP $(\%)$ & p-value \\
\cline { 3 - 5 } & $54(62.8)$ & $18(33.3)$ & $18(33.3)$ & $18(33.3)$ & 0.0138 \\
$<50$ & $32(37.2)$ & $17(53.1)$ & $2(6.25)$ & $13(40.6)$ &
\end{tabular}

Fourteen breast cancer patients were not included as their ages were unknown. SNP, single nucleotide polymorphism; RR, Arg/Arg; RP, Arg/Pro; PP, Pro/Pro.

genotype was highly frequent in young breast cancer patients as compared with older patients (33.3 and $6.25 \%$, respectively). The PP form appeared in 33.3 and $40.6 \%$ of the young and old patients, respectively. This indicates that while the RR form is more prevalent in older breast cancer patients, the RP form is less frequent in these patients. These results are statistically significant (SNP72 vs. the age of Saudi breast cancer patients, $\mathrm{p}=0.0138$ ). The RP genotype is associated with the early onset of breast cancer, whereas the RR genotype is associated with late onset of the disease.

The p53 codon 47 polymorphism reveals no association with sporadic breast cancer in Saudi patients. The codon 47 SNP is located in exon 4 . This codon encodes either proline, when the sequence is CCG, or serine, when the sequence is TCG. In this study, all sequenced DNA (100\%) was found to carry the wild-type sequence CCG (proline) at codon 47.

\section{Discussion}

The association between the p53 codon 72 polymorphism and breast cancer risk was studied in different populations, yielding conflicting results (14). In the present study, we investigated this association in the Saudi population and found that, while the RP genotype is the most abundant among healthy women $(60.19 \%)$, its prevalence is the lowest among women with breast cancer $(25 \%)$, suggesting that this genotype is a protection factor against breast cancer in the Saudi population. By contrast, the frequency of the p53 RR form was higher in the breast cancer patients than in the controls, with 39 and $17.6 \%$, respectively, suggesting that the RR genotype is a potential risk factor for breast tumorigenesis among Saudi women. A statistically significant association was found between SNP72 and breast cancer risk in the Saudi population $(\mathrm{p}=0.0001)$. These results are in agreement with other published data, which reported a marked association between the RR form of SNP72 and breast cancer risk (15-16). Similar results have also been reported regarding cervical cancer (17). Furthermore, Alawadi et al and a recent meta-analysis showed the PP genotype to be associated with decreased breast cancer risk among the Kuwaiti and Indian populations, respectively $(14,18)$. However, Dumont et al found that the proline variant is associated with an increased risk of breast cancer (19). By contrast, other studies did not observe any association between the TP53 codon 72 polymorphism and breast cancer risk (20-22). Similarly, the most recent meta-analysis indicated that the codon 72 polymorphism may not be associated with breast cancer risk in a Caucasian population. However, previous meta-analyses concerning the p53 codon 72 polymorphism also revealed conflicting results (23-26).

The precise reasons for this discrepancy remained to be elucidated $(8,11)$. One possibility is that the effect of this polymorphism varies according to the genetic background of the studied population. Francisco et al suggested that ethnicity and histological and anatomical sites modulate the penetrance of R72P in cancer susceptibility (26). The other possibility includes the size of the studied population and stringent levels of statistical significance (7). The presence of unknown variables that are not presently controlled in such studies may also be a reason for discrepancy.

Results of this study have shown a statistically significant association between the TP53 codon 72 polymorphism and the age of Saudi breast cancer patients $(\mathrm{p}=0.0138)$. The RP form is more frequent in young breast cancer patients compared with older patients (33.3 and 6.25\%, respectively). Previously, it was reported that there is an association between the codon 72 polymorphism and the early onset of cancer, particularly with the PP form of SNP72 (11). However, no study exists regarding an association between this polymorphism and breast cancer. By contrast, certain studies found no association between this SNP and the age of onset of breast cancer in the Chinese (22), Japanese (10) and Iranian populations (20). The reason for this discrepancy may be population-dependent, for example other genetic or environmental factors.

Findings of recent studies have shown that the rare TP53 codon 47 polymorphism may also be involved in cancer risk, cancer progression and the effectiveness of therapy. However, only small numbers of studies have been performed on SNP47 and its association with cancer due to the low frequency of this polymorphism (11). In the present study, it was found that $100 \%$ of the Saudi women involved in the study carried the wild-type sequence CCG (proline) at codon 47 of the TP53 gene. Similar results were reported in the Kuwaiti population, wherein the the codon 47 variant was detected in only one patient and none of the controls (18). Therefore, the rare sequence TCG (serine) of SNP47, which is thought to reduce the p53 apoptosis ability 5-fold as compared with the wild-type p53 proline 47 (7), is extremely rare or non-existent in the Saudi Arab population. This indicates that there is no association between the TP53 SNP47 and breast cancer in the Saudi population.

In conclusion, findings of the present study have shown that the RR form of SNP72 is a potential risk factor, while the RP 
form is a protection factor against breast cancer among Saudi women. Moreover, the RP form is more prevalent among young ( $<50$ years) Saudi breast cancer patients compared with older ( $\geq 50$ years) patients (33 and $6 \%$, respectively; $p=0.0138$ ). These results indicate that SNP72 is markedly associated with both the risk and early onset of breast cancer in the Saudi population. We have also found that $100 \%$ of the Saudi women in this study carried the wild-type proline at codon 47 . Thus, the codon 72 polymorphism is markedly associated with the risk and early onset of breast cancer, whereas the codon 47 polymorphism has no impact on breast cancer in the Saudi population.

\section{Acknowledgements}

We are grateful to KACST for their financial help. We also thank KFSH\&RC administration as well as the Training and Education and ORA offices for their continuous help. This study was performed under the RAC proposal \#2040037 and KACST \#LPG 10-9.

\section{References}

1. Jemal A, Bray F, Center MM, et al: Global cancer statistics. CA Cancer J Clin 61: 69-90, 2011.

2. Ezzat AA, Ibrahim EM, Raja MA, et al: Locally advanced breast cancer in Saudi Arabia: high frequency of stage III in a young population. Med Oncol 16: 95-103, 1999.

3. Elkum N, Dermime S, Ajarim D, et al: Being 40 or younger is an independent risk factor for relapse in operable breast cancer patients: the Saudi Arabia experience. BMC Cancer 7: 222, 2007.

4. Greenblatt MS, Bennett WP, Hollstein M and Harris CC: Mutations in the p53 tumor suppressor gene: clues to cancer etiology and molecular pathogenesis. Cancer Res 54: 4855-4878, 1994.

5. Levine AJ and Oren M: The first 30 years of p53: growing ever more complex. Nat Rev Cancer 9: 749-758, 2009.

6. Pietsch EC, Humbey $\mathrm{O}$ and Murphy ME: Polymorphisms in the p53 pathway. Oncogene 25: 1602-1611, 2006.

7. Whibley C, Pharoah PD and Hollstein M: p53 polymorphisms: cancer implications. Nat Rev Cancer 9: 95-107, 2009.

8. Hartmann W, Digon-Sontgerath B, Koch A, et al: Phosphatidylinositol 3'-kinase/AKT signaling is activated in medulloblastoma cell proliferation and is associated with reduced expression of PTEN. Clin Cancer Res 12: 3019-3027, 2006.

9. Wei M, Grushko TA, Dignam J, et al: BRCA1 promoter methylation in sporadic breast cancer is associated with reduced BRCA1 copy number and chromosome 17 aneusomy. Cancer Res 65: 10692-10699, 2005.
10. Toyama T, Zhang Z, Nishio M, et al: Association of TP53 codon 72 polymorphism and the outcome of adjuvant therapy in breast cancer patients. Breast Cancer Res 9: R34, 2007.

11. Murphy ME: Polymorphic variants in the p53 pathway. Cell Death Differ 13: 916-920, 2006.

12. Felley-Bosco E, Weston A, Cawley HM, Bennett WP and Harris CC: Functional studies of a germ-line polymorphism at codon 47 within the p53 gene. Am J Hum Genet 53: 752-759, 1993.

13. Li X, Dumont P, Della Pietra A, Shetler C and Murphy ME: The codon 47 polymorphism in p53 is functionally significant. J Biol Chem 280: 24245-24251, 2005.

14. He XF, Su J, Zhang Y, et al: Association between the p53 polymorphisms and breast cancer risk: meta-analysis based on case-control study. Breast Cancer Res Treat 130: 517-529, 2011.

15. Buyru N, Tigli H and Dalay N: P53 codon 72 polymorphism in breast cancer. Oncol Rep 10: 711-714, 2003.

16. Papadakis EN, Dokianakis DN and Spandidos DA: p53 codon 72 polymorphism as a risk factor in the development of breast cancer. Mol Cell Biol Res Commun 3: 389-392, 2000.

17. Jee SH, Won SY, Yun JE, et al: Polymorphism p53 codon-72 and invasive cervical cancer: a meta-analysis. Int J Gynaecol Obstet 85: 301-308, 2004

18. Alawadi S, Ghabreau L, Alsaleh M, et al: P53 gene polymorphisms and breast cancer risk in Arab women. Med Oncol 28: 709-715, 2011.

19. Dumont P, Leu JI, Della Pietra AC III, George DL and Murphy M: The codon 72 polymorphic variants of $p 53$ have markedly different apoptotic potential. Nat Genet 33: 357-365, 2003

20. Khadang B, Fattahi MJ, Talei A, Dehaghani AS and Ghaderi A: Polymorphism of TP53 codon 72 showed no association with breast cancer in Iranian women. Cancer Genet Cytogenet 173: 38-42, 2007.

21. Tommiska J, Eerola H, Heinonen M, et al: Breast cancer patients with p53 Pro72 homozygous genotype have a poorer survival. Clin Cancer Res 11: 5098-5103, 2005.

22. Xu Y, Yao L, Zhao A, et al: Effect of p53 codon 72 genotype on breast cancer survival depends on p53 gene status. Int J Cancer 122: 2761-2766, 2008

23. Zhang Z, Wang M, Wu D, et al: P53 codon 72 polymorphism contributes to breast cancer risk: a meta-analysis based on 39 case-control studies. Breast Cancer Res Treat 120: 509-517, 2010.

24. Hu Z, Li X, Yuan R, Ring BZ and Su L: Three common TP53 polymorphisms in susceptibility to breast cancer, evidence from meta-analysis. Breast Cancer Res Treat 120: 705-714, 2010.

25. Ma Y, Yang J, Liu Z, et al: No significant association between the TP53 codon 72 polymorphism and breast cancer risk: a metaanalysis of 21 studies involving 24,063 subjects. Breast Cancer Res Treat 125: 201-205, 2011.

26. Francisco G, Menezes PR, Eluf-Neto J and Chammas R: Arg72Pro TP53 polymorphism and cancer susceptibility: A comprehensive meta-analysis of 302 case-control studies. Int J Cancer 129: 920-930, 2011. 